\title{
Pomegranate Fruit Diseases Identification and Grading
}

\author{
D. Gaikwad ${ }^{1}$, K. Karande ${ }^{2}$ and H. Deshpande ${ }^{3}$ \\ ${ }^{1}$ Student, SKN Sinhgad college of Engineering, Korti Pandharpur 413304 Dist-Solapur, Maharashtra \\ 2 Professor, SKN Sinhgad college of Engineering, Korti Pandharpur 413304 Dist-Solapur, Maharashtra \\ ${ }^{3}$ Assistant Professor, SKN Sinhgad college of Engineering, Korti Pandharpur 413304 Dist-Solapur, Maharashtra \\ \{dhanshri712@gmail.com; kailashkarande@yahoo.co.in; hrushikesh102@gmail.com\}
}

\begin{abstract}
India is one of the well-known country in world, in the area of pharmacy specially food horticulture. India produces nearly 5.00 lacks tones/annum pomegranate. Fruit gradation is one of the most vital parts in fruit horticulture. The project design presented by this paper is from same problematic area. In our project design we developed systems which classify diseases affecting pomegranates using K-means clustering and SVM techniques. We are classifying the different pomegranate variety in accordance with their diseases. This paper deals with pomegranate grading and identification of disease system with judging parameters. The specialty of design is it creates a model which helps to decide appropriate criteria for healthy fruit. This project design is acts as advance system model in Indian horticulture for deciding ranges of mean, variance, entropy values by which the quality of fruit is decided. These parameters are judging parameters of our project design.
\end{abstract}

Keywords: Pomegranate, K-means, SVM (support vector machine), mean, variance

\section{Introduction}

Fruit horticulture is the backbone of agriculture development of any country. The quality of fruit is decided by two factors, one is the weight, nutrients and another one is detection of diseases. Fruit diseases or fruit plant diseases cause major production and economic losses in agriculture industries. In India according to survey of APEDA \& DGCIS annual report nearly 5lacks tones pomegranate production takes place where as in total state Maharashtra is the most leading state in production of pomegranate. In Maharashtra $98.9 \%$ area is under pomegranate horticulture. In the domain of agriculture no sensor is available which deals with real time diagnosis and analysis of healthy condition of fruit. The project design implemented by this paper focuses on various diseases category affecting on pomegranate these are Alternaria fruit spot, cercospora fruit spot, bacterial blight, fruit rot. According to survey of economic time; in 2007-2008, 3500 metric tons of fruit figure drops down to 33,400 in 2009-2010. The main reason for declining the production is infection by Bacterial Blight Disease (Telya-in Marathi). Our project deals with detection of disease according to its characteristics and validates the fruit quality for healthy fruit. This paper is divided into 4 sections as - 1.Introduction, 2 . Dataset, 3. Methodology, 4. Experimental results, 5. Conclusions.

\section{Dataset}

There is no any standard database is available for this work. So we collect the pomegranate fruit images from NRCP ( National Research Center on Pomegranate, Solapur) and also some images are capture from camera by realtime farming. In this research work we are using about 40 images of pomegranate fruit which are classified by proposed method.Some diseased images are shown below in Fig.1.

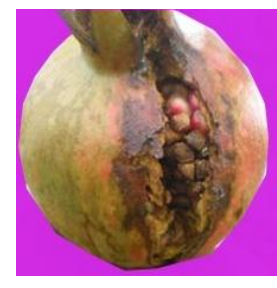

(a) Bacterial blight

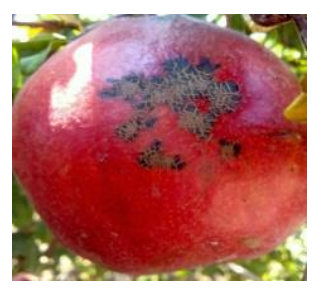

(b) Alternaria fruit spot

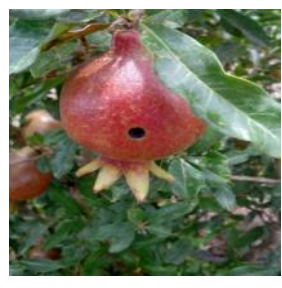

(c) Cercospora Fruit Spot

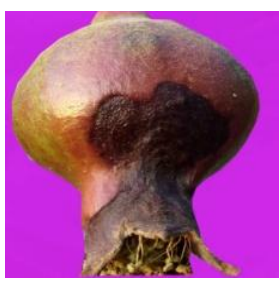

(d) Fruit Rot

Fig.1. Pomegranate fruit diseases images

B. Iyer, S. Nalbalwar and R.Pawade(Eds.)

ICCASP/ICMMD-2016. Advances in Intelligent Systems Research.

Vol. 137, Pp. 689-696.

(C) 2017- The authors. Published by Atlantis Press 
Above four diseases shows different symptoms. Depending upon these symptoms each disease is classified into their respective diseases category.

\section{Methodology}

Flow of the proposed work is given below, at first the diseases images of pomegranate fruit given as input to the system. This image is pre-processed. As we are interested to identify the type of diseases the image is then segmented by using K-means clustering algorithm. Then features are extracted from segmented cluster containing diseases part. Classification is done by using multiclass SVMs,

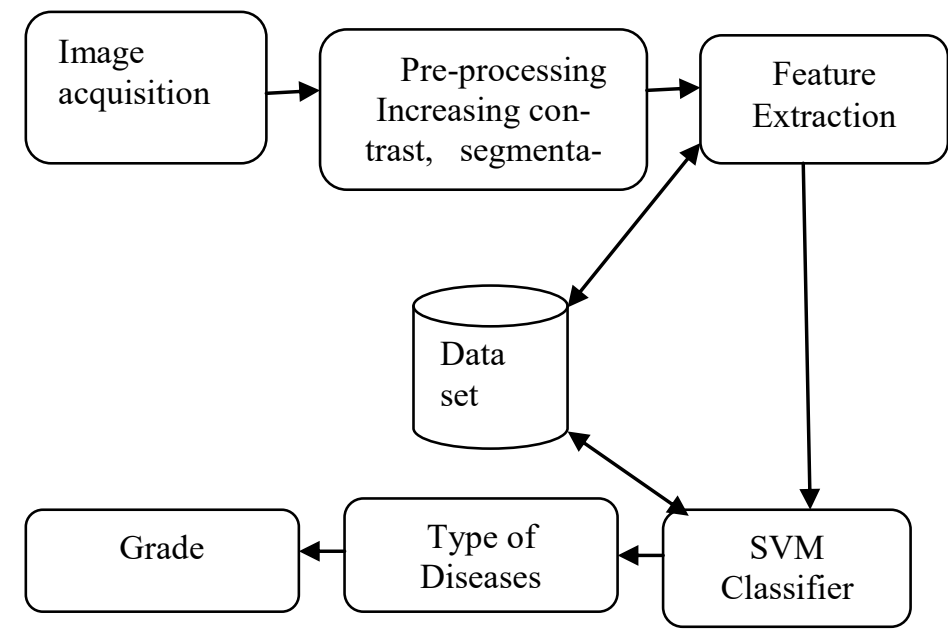

Fig.2. Flowchart of the proposed work

\subsection{Image Acquisition}

The first phase of any vision system is the image acquisition stage. After collecting the images, different methods of processing can be applied to the image to achieve proposed tasks.

\subsection{Pre-processing}

After getting images of pomegranate fruit next step is image pre-processing. Here first we increase the contrast of the image so it will become more meaningful for further use. Healthy fruit image is as shown below,

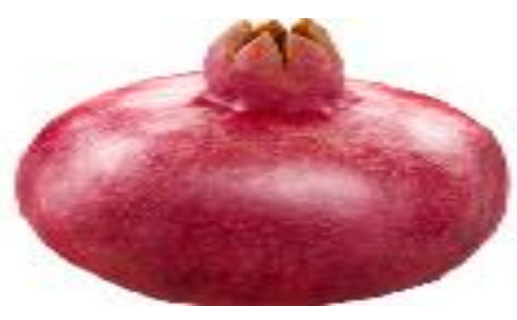

Fig.3. Healthy Pomegranate fruit image

After increasing its contrast it will be look like as shown below,

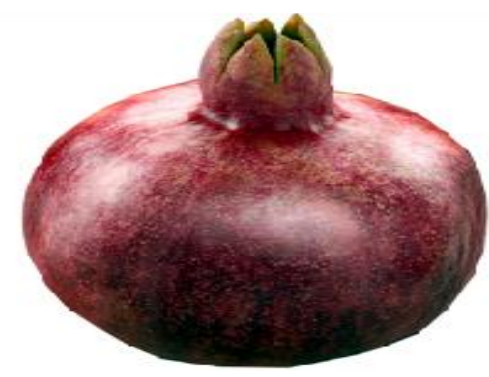

Fig.4. Enhanced image of Pomegranate fruit. 


\subsection{Image Segmentation}

Image segmentation is used to partition the diseased part and healthy part of the pomegranate fruit. K-means clustering algorithm is used to form the cluster to find diseases part. It forms the three clusters as shown in Fig.5 below.

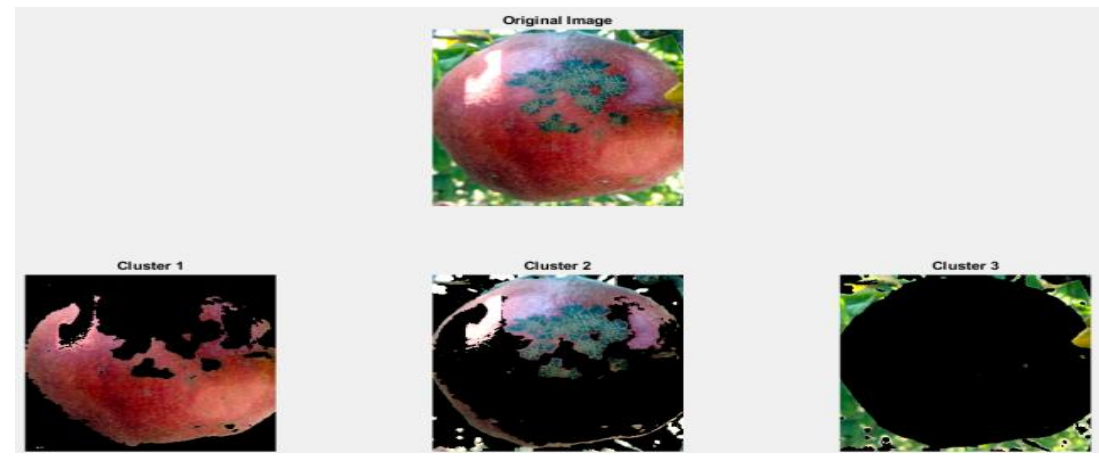

Fig.5. Segmented results of diseases Alternaria fruit spot

\subsection{Feature extraction}

Feature extraction is the procedure to opt for the important characteristics of an image. Transforming the input data into the set of features is called feature extraction. Thirteen revealing features are extracted from the pomegranate fruit image are Mean, Variance, Entropy, RMS value, Standard deviation, Smoothness, Kurtosis, Skewness, IDM, Contrast, Correlation, Energy, Homogeneity. These are the key features which gives specific range for each disease.

\subsection{Classification}

Support vector machine (SVM) concept is used for classification. Support vector machines (SVMs) were initially intended for binary classification. A number of methods have been projected where usually we create a multiclass classifier by combining some binary classifiers. Some authors also wished-for methods that consider all classes at once. A support vector machine constructs a set of hyper planes in a high- or infinite-dimensional space, which can be used for classification, Multiclass SVM aims to give labels to instances by using support vector machines, where the labels are drained from a finite set of several essentials. In this proposed work multiclass SVM classify the pomegranate diseases images into the respective diseases category.

\section{Experimental Results.}

The each disease shows specific symptoms.

\section{1. Alternaria fruit spot.}

As Alternaria fruit spot shows the reddish brown spot appear on the fruit. We have taken two sample image of Alternaria fruit spot diseases image.

Table1. Parameters calculated for diseases Alternaria fruit spot.

\begin{tabular}{llllllc}
\hline Parameter & Mean & S.D & Entropy & RMS & Variance & smoothness \\
\hline Sample 1 & 44.80 & 73.5 & 3.52 & 0.902 & 5068.25 & 1 \\
Sample 2 & 45.44 & 78.4 & 3.95 & 8.947 & 4618.29 & 1 \\
\hline Parameter & Kurtosis & Skewness & IDM & Contrast & Correlation & Energy \\
\hline Sample 1 & 3.92 & 1.47248 & 255 & 0.530178 & 0.5301 & 0.437874 \\
Sample 2 & 4.44 & 1.68273 & 255 & 0.633686 & 0.6336 & 0.413304 \\
\hline Parameter & Homogeneity & & & & \\
\cline { 1 - 1 } Sample 1 & 0.936429 & & & & & \\
Sample 2 & 0,924113 & & & & &
\end{tabular}


The thirteen features give the above range for diseases Alternaria fruit spot. From this feature the diseases classified is shown below Fig 7, 8

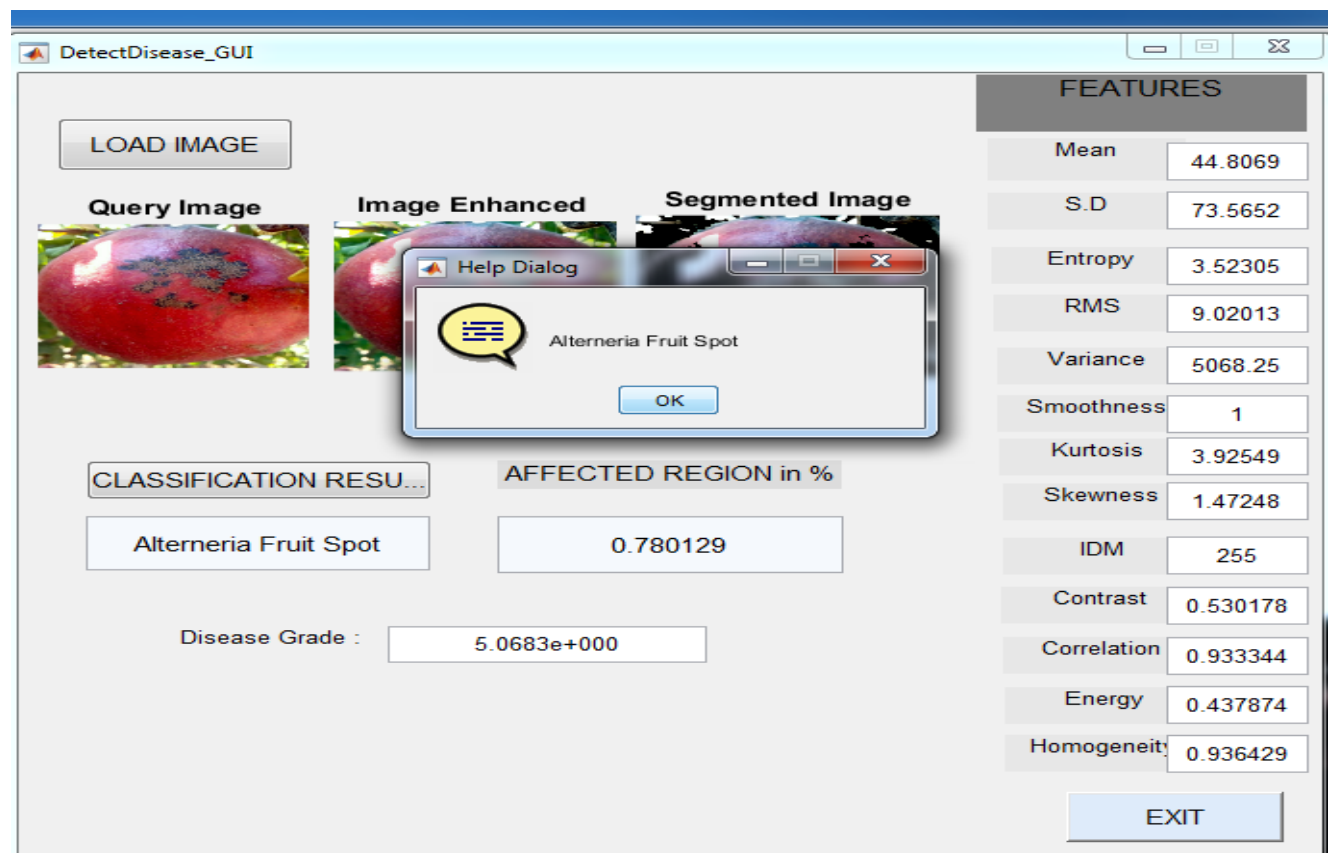

Fig.7. Sample 1: Result of Alternaria fruit spot disease.

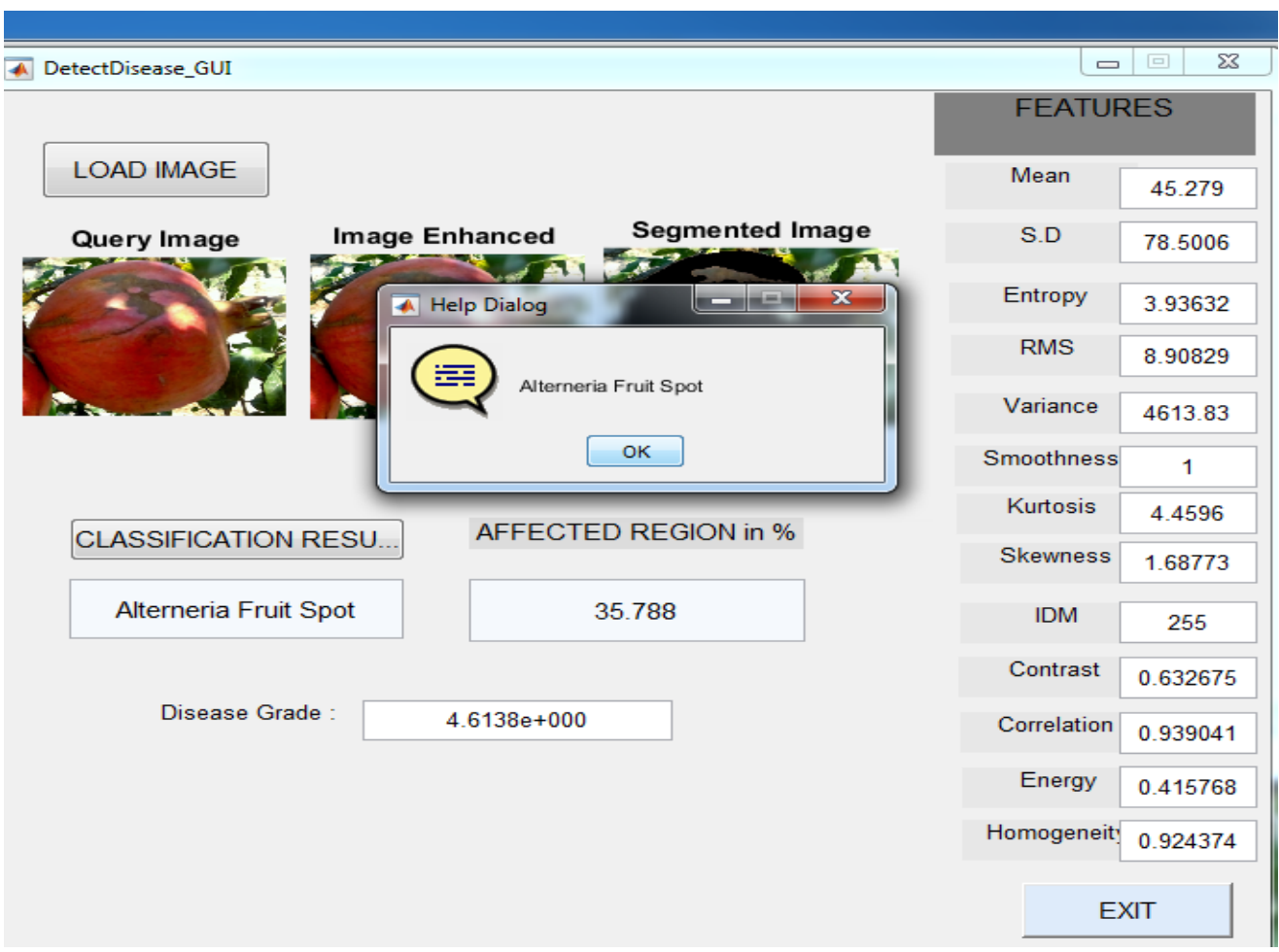

Fig.8. Sample 2: Result of Alternaria fruit spot disease. 


\subsection{Bacterial blight.}

The features extracted from this disease are given below

Table 2. Parameters calculated for the diseases Bacterial blight

\begin{tabular}{llllllc}
\hline Parameter & Mean & S.D & Entropy & RMS & Variance & smoothness \\
\hline Sample 1 & 12.6551 & 36.9434 & 1.4761 & 3.91582 & 1024.52 & 1 \\
Sample 2 & 17.2062 & 47.2358 & 1.9067 & 4.66964 & 1764.51 & 1 \\
\hline Parameter & Kurtosis & Skewness & IDM & Contrast & Correlation & Energy \\
\hline Sample 1 & 10.6522 & 2.94946 & 255 & 0.15781 & 0.928083 & 0.758036 \\
Sample 2 & 11.6005 & 3.0078 & 255 & 0.25239 & 0.918169 & 0.706573 \\
\hline Parameter & Homogeneity & & & & \\
\hline Sample 1 & 0.973409 & & & & & \\
Sample 2 & 0.968913 & & & & &
\end{tabular}

From this features the diseases classify is the Bacterial blight. The result shown for Bacterial blight is given below,

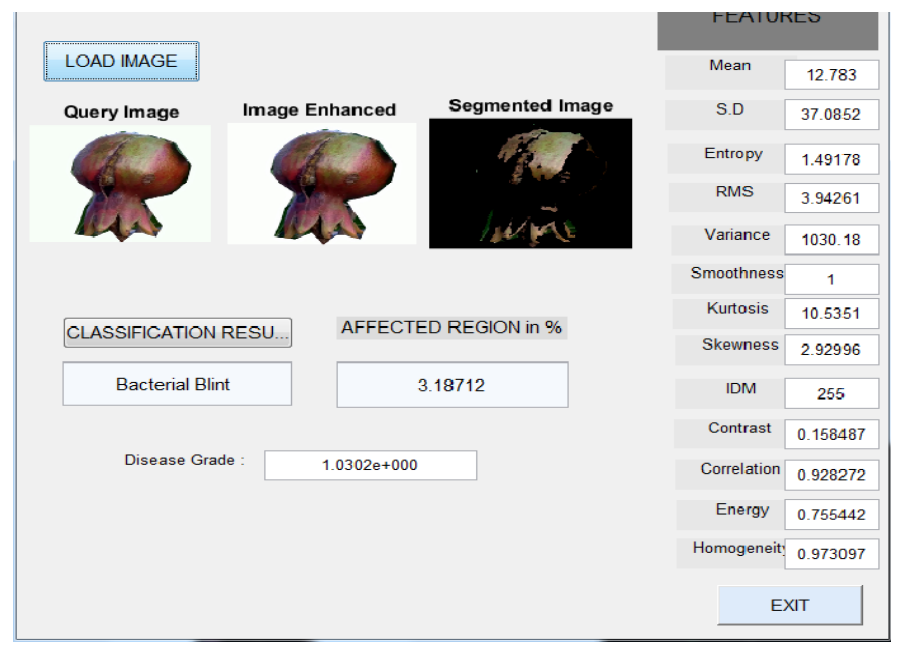

Fig.9. Sample 1: Result of Bacterial blight disease

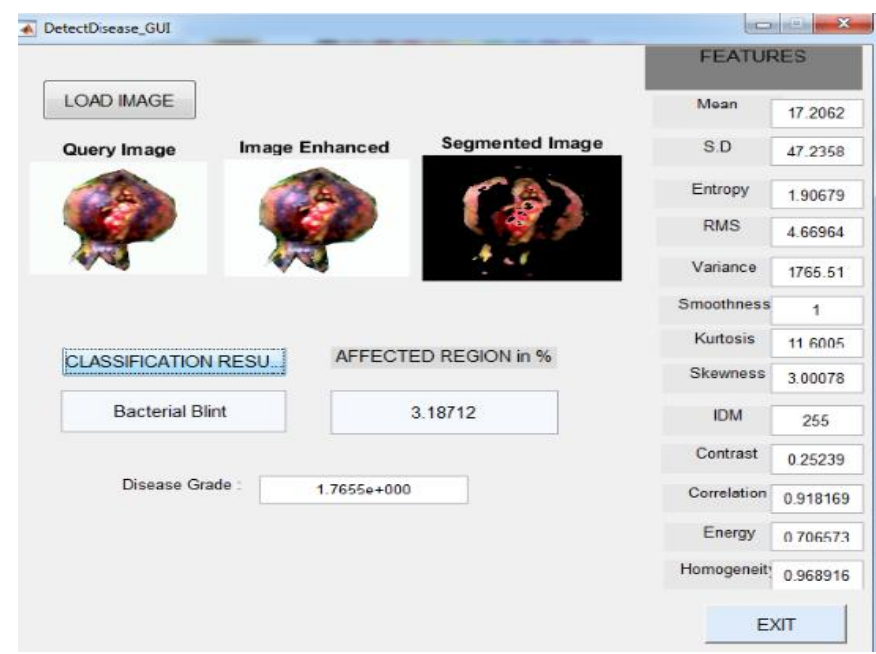

Fig.10. Sample 2: Result of Bacterial blight disease. 


\subsection{Cercospora fruit spot}

Here we take the two examples of cercospora fruit spot diseases images. The value of parameters calculated from these diseases images are given below,

Table 3. Parameters calculated for the diseases Cercospora fruit spot

\begin{tabular}{llllllc}
\hline Parameter & Mean & S.D & Entropy & RMS & Variance & smoothness \\
\hline Sample 1 & 48.3948 & 66.679 & 4.5932 & 10.6832 & 4211.31 & 1 \\
Sample 2 & 48.2433 & 66.648 & 4.5804 & 10.6643 & 4210.73 & 1 \\
\hline Parameter & Kurtosis & Skewness & IDM & Contrast & Correlation & Energy \\
\hline Sample 1 & 3.85544 & 1.32571 & 255 & 0.97340 & 0.863348 & 0.298356 \\
Sample 2 & 3.86709 & 1.33027 & 255 & 0.97121 & 0.863415 & 0.299887 \\
\hline Parameter & Homogeneity & & & & \\
\hline Sample 1 & 0.895272 & & & & \\
Sample 2 & 0,895487 & & & &
\end{tabular}

This is the range of features for cercospora fruit spot.

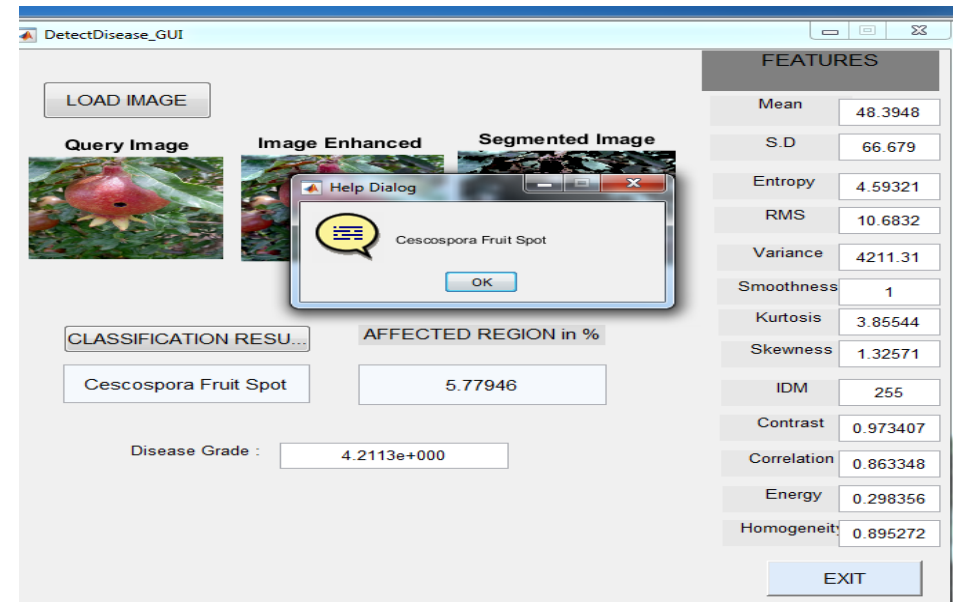

Fig.11. Sample 1: Result of cercospora fruit spot disease

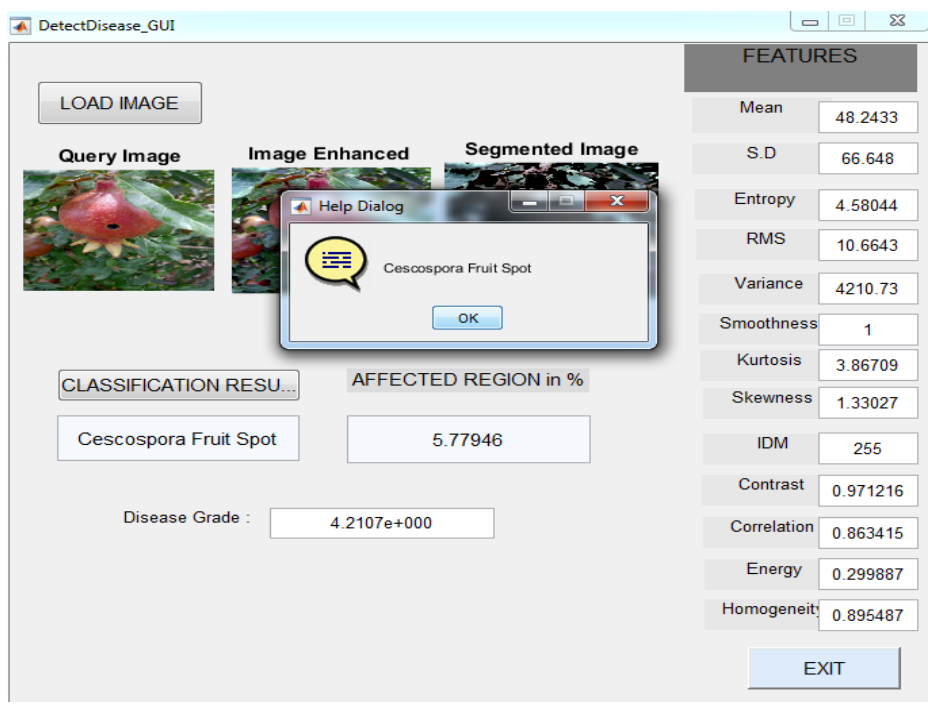

Fig.12. Sample 2: Result of cercospora fruit spot disease. 


\subsection{Fruit rot.}

Let's take two example of fruit rot. The features calculated for this diseases is given below,

Table 4. Parameters calculated for the diseases Fruit rot

\begin{tabular}{lllclcc}
\hline Parameter & Mean & S.D & Entropy & RMS & Variance & smoothness \\
\hline Sample 1 & 147.104 & 116.684 & 3.7922 & 13.0698 & 12090.6 & 1 \\
Sample 2 & 31.4058 & 59.6635 & 3.4861 & 6.92884 & 2480.09 & 1 \\
\hline Parameter & Kurtosis & Skewness & IDM & Contrast & Correlation & Energy \\
\hline Sample 1 & 1.21385 & 0.33242 & 255 & 0.25162 & 0.9888235 & 0366311 \\
Sample 2 & 5.68068 & 1.93638 & 255 & 0.39947 & 0.932955 & 0.536427 \\
\hline Parameter & Homogeneity & & & & & \\
\hline Sample 1 & 0.98268 & & & & & \\
Sample 2 & 0.970927 & & & & &
\end{tabular}

From above features the diseases is classified into the fruit rot. Results are shown below,

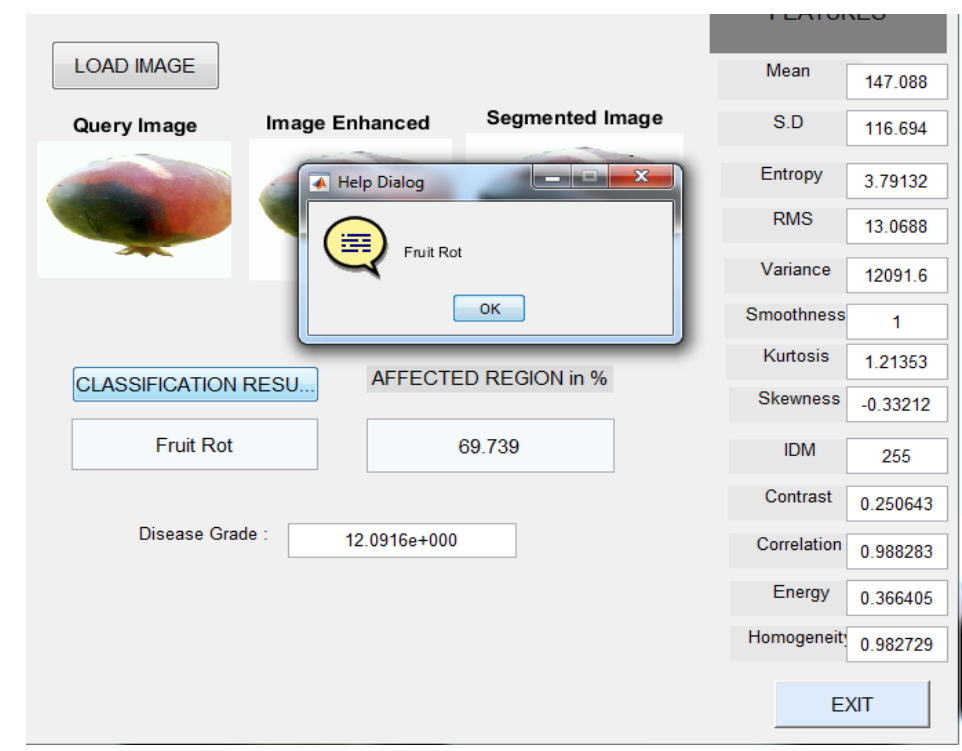

Fig.13. Sample 1: Result of fruit rot disease.

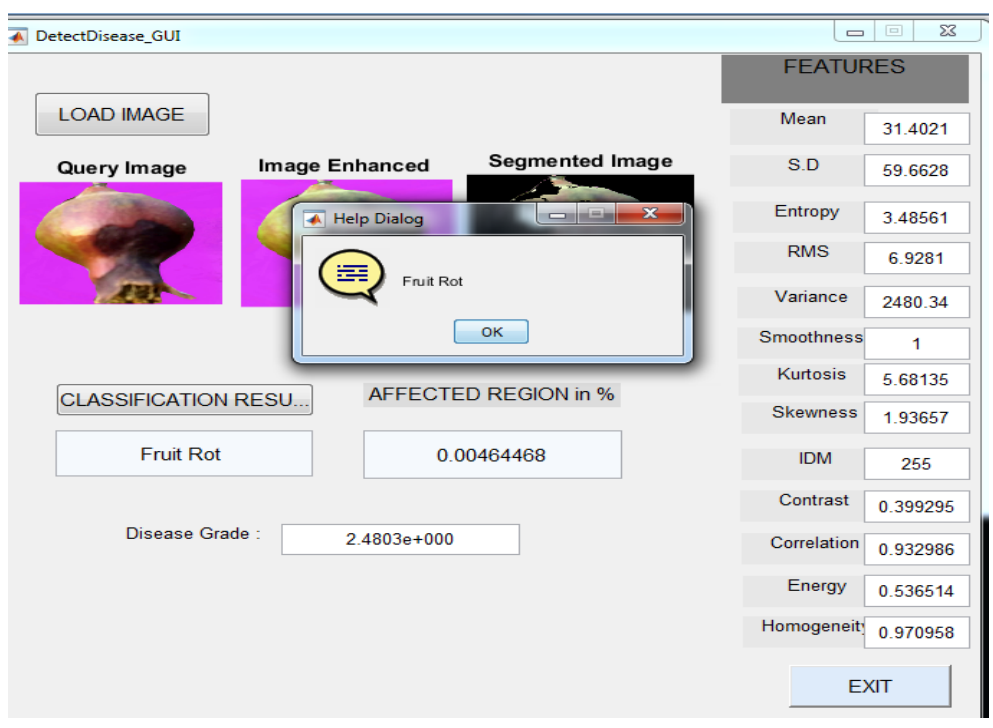

Fig.14. Sample 2: Result fruit rot disease. 
It is observe that the pomegranate diseases images are classified into the respective diseases category. Four diseases Alternaria fruit spot, Bacterial blight, Cercospora fruit spot, Fruit rot and Healthy fruit images are identified correctly.

\section{Conclusions.}

Automation in agriculture is very important to help the farmers. This paper is useful for detecting the four diseases on pomegranate fruit and also grading the fruit depending upon their diseased portion. Feature extraction and SVM multiclass classifier is the important steps in this work. Range of the dataset is created from the analysis of the features value. From result it can be seen that the all four diseases are classified very correctly.

\section{References}

[1] Tejal Deshpande, Sharmila Sengupta, K. S. Raghuvanshi , "Grading \& Identification of Disease in Pomegranate Leaf and Fruit," Vol.5(3), 2014, 4638-4645.

[2] Monika Jhuria, Ashwani Kumar, Rushikesh Borse, "Image Processing For Smart Farming: Detection of Diseases and Fruit Grading," IEEE ICIIP, pp.521-526, 2013.

[3] Sindhuja Sankarana, Ashish Mishra Reza Ehsania, Cristina Davisb, "A review of advanced techniques for detecting plant diseases," Computers and Electronics in Agriculture, vol. 72, pp.1-13, 2010.

[4] Usmail Kavdir,Daniel E. Guyer, “Apple Grading Using Fuzzy Logic”, Turk J Agric (2003), 375-382

[5] C.C.Teoh and A.R Mohd Syaifudin,"Image processing and analysis techniques for estimating weights of Chikanan mangoes "J. Trop.Agric.and fd. Sc., vol35 (1), pp.183-190, 2007.

[6] Sanjeev S Sannakki1, Vijay S Rajpurohit, V B Nargund, ArunKumar R, Prema S Yallur, "Leaf Disease Grading by MachineVision and Fuzzy Logic", Int. J. Comp. Tech. Appl., Vol 2 (5),1709-1716, 2011.

[7] Boaz lerner, Hugo Guterman, mayer Aladjem,Its'hak Dinstein," A comparative study of neural network based feature extraction paradigm," pattern Recognition Letters, vol.20, pp.7-14, 1999.

[8] Rafael C, Gonzalez, Richard E. Woods, "Digital Image Processing", second edition,2005.

[9] H.D. Cheng, X.H. Jiang, Y. Sun, Jingli Wang," Color image segmentation: advances and prospects," Pattern Recognition 34, PP.2259-2281, 2001.

[10] Anand.H.Kulkarni1, Ashwin Patil R. K.," Applying image processing technique to detect plant diseases" Vol.2, Issue.5, Sep-Oct. 2012 pp-3661-3664. 